\title{
EXAMPLE OF A NON-LOG-CONCAVE DUISTERMAAT-HECKMAN MEASURE
}

\author{
YAEL KARSHON
}

\begin{abstract}
We construct a compact symplectic manifold with a Hamiltonian circle action for which the Duistermaat-Heckman function is not log-concave. This provides a counter-example to a conjecture of Ginzburg and Knudsen.
\end{abstract}

\section{Introduction}

Let $T$ be a torus and $\mathfrak{t}$ its Lie algebra. Let $(M, \omega)$ be a symplectic manifold with an action of $T$ and with a moment map

$$
\Phi: M \rightarrow \mathfrak{t}^{*}
$$

Recall, this means that for every $\xi \in \mathfrak{t}$, if $\xi_{M}$ is the corresponding vector field on $M, \iota\left(\xi_{M}\right) \omega=-d\langle\Phi, \xi\rangle$.

Liouville measure on $M$ associates to an open set $U$ the measure $\int_{U} \omega^{n}$ where $n$ is half the dimension of the manifold and where we integrate with respect to the symplectic orientation. The Duistermaat-Heckman measure $[\mathrm{DH}]$ on $\mathfrak{t}^{*}$ is the push-forward of Liouville measure via the moment map $\Phi$. If $T$ acts effectively, the Duistermaat-Heckman measure is absolutely continuous with respect to Lebesgue measure, and the density function on $\mathfrak{t}^{*}$ is called the Duistermaat-Heckman function.

Ginzburg and Knudsen conjectured [Gi, Kn] that for any Hamiltonian torus action on a compact manifold, the Duistermaat-Heckman function is log-concave, i.e., its logarithm is concave. This conjecture was supported by the following evidence.

(1) The image of the moment map is a convex polytope [GS, At]. If the dimension of $T$ is half the dimension of $M$ and $T$ acts effectively, the Duistermaat-Heckman function is equal to one on this polytope and zero outside [De]. This function is log-concave, i.e., its logarithm is concave.

Received June 19, 1996.

The author is partially supported by NSF grant DMS-9404404.

dg-ga/9606007. 
(2) If we restrict a Hamiltonian action of a torus $T$ to a subtorus $H$, the Duistermaat-Heckman function for $H$ evaluated at a point $\alpha \in$ $\mathfrak{h}^{*}$ is equal to the integral of the Duistermaat-Heckman function for $T$ over the fiber $\pi^{-1}(\alpha)$ of the linear projection $\pi: \mathfrak{t}^{*} \rightarrow \mathfrak{h}^{*}$. If we start from a log-concave function on $\mathfrak{t}^{*}$, we get in this way a log-concave function on $\mathfrak{h}^{*}[\mathrm{Pr}$, Theorem 6].

The log-concavity conjecture was proved for circle actions on four manifolds by the author [Ka, $\S 2.6]$, for coadjoint orbits in classical groups and for arbitrary projective algebraic varieties by A. Okounkov [Ok1, Ok2], and for arbitrary Kähler manifolds by W. Graham [Gr]. In this note we construct a counterexample to the conjecture; we construct a Hamiltonian circle action on a compact symplectic 6-manifold for which DuistermaatHeckman function is not log-concave. Our manifold does not admit an equivariant Kähler structure; this follows from Graham's result.

The construction came from investigating an example of Dusa McDuff of a 6 -manifold with a circle valued moment map [MD]. I use her notation wherever possible.

Our conventions regarding factors of $2 \pi$ etc. are irrelevant and will not be made explicit.

\section{The construction}

Let $T^{4}$ be the four dimensional torus with periodic coordinates $x_{i}, 1 \leq$ $i \leq 4$, and let $\sigma_{i j}=d x_{i} \wedge d x_{j}$ and $\sigma_{1234}=d x_{1} \wedge d x_{2} \wedge d x_{3} \wedge d x_{4}$. Let $L$ be a complex Hermitian line bundle over $T^{4}$ with Chern class $\left[-\sigma_{14}-\sigma_{32}\right]$. Let $\Theta$ be a connection one-form with curvature $-\sigma_{14}-\sigma_{32}$. By this we mean that $\Theta$ is defined on $L$ outside the zero section, that the restriction of $\Theta$ to a fiber of $L$ is $d \theta$ in polar coordinates on the fiber, and that $d \Theta$ is the pullback of $-\sigma_{14}-\sigma_{32}$ via the bundle map $L \rightarrow T^{4}$. Denote by the same letters $\sigma_{i j}, \sigma_{1234}$ the pullbacks of these forms to $L$. Let the function $\Phi: L \rightarrow \mathbb{R}$ be the norm squared, with respect to the fiberwise Hermitian metric on $L$. Consider the two-form

$$
\omega=\sigma_{12}+\sigma_{34}+(2-\Phi) \sigma_{14}+(3-\Phi) \sigma_{32}+d \Phi \wedge \Theta
$$

on $L$ minus its zero section. It is easy to check that $\omega$ is closed and that its top power is

$$
\omega^{3}=6(1+(2-\Phi)(3-\Phi)) \sigma_{1234} \wedge d \Phi \wedge \Theta .
$$

Since $\sigma_{1234} \wedge d \Phi \wedge \Theta \neq 0$ and since the function $(1+(2-s)(3-s))$ is always positive, $\omega$ is symplectic.

The circle group acts on $L$ by fiberwise rotation. Let $\xi$ be the generating vector field. From (1) it is clear that $\iota(\xi) \omega=-d \Phi$, so $\Phi$ is a moment map 
for the circle action. The Duistermaat-Heckman function is a constant positive multiple of the function

$$
\rho(s)=1+(2-s)(3-s) .
$$

This function decreases for $0<s<2.5$ and increases for $2.5<s<\infty$, so it is not log-concave.

To make a compact example out of our noncompact one, we perform Lerman's "symplectic cutting" [Le]: choose any two numbers, $0<A<2.5$ and $2.5<B<\infty$. Cutting produces a compact symplectic manifold $(M, \omega)$ with a circle action and a moment map $\Phi: M \rightarrow[A, B]$ such that the preimages in $M$ and in $L$ of the open interval $(A, B)$ are equivariantly symplectomorphic. Consequently, the Duistermaat-Heckman functions are the same: for the compact manifold $M$ we get the function (2) restricted to the interval $A \leq s \leq B$, and this function is not log-concave.

\section{Acknowledgement}

I wish to thank E. Lerman for suggesting to publish this example, Y. Peres for advising me on convex sets and log-concave functions, and Dror B-N., E. Lerman, and the anonymous referee for commenting on the manuscript.

\section{References}

[At] M. Atiyah, Convexity and commuting hamiltonians, Bull. London Math. Soc. 14 (1982), 1-15.

[De] T. Delzant, Hamiltoniens périodiques et image convexe de l'application moment, Bull. Soc. Math. France 116 (1988), 315-339.

$[\mathrm{DH}]$ J. J. Duistermaat and G. J. Heckman, On the variation in the cohomology of the symplectic form of the reduced phase space, Invent. Math. 69 (1982), 259-269.

[Gi] V. Ginzburg (University of Chicago), August 1994.

[Gr] W. Graham, Logarithmic convexity of push-forward measures, Invent. Math. 123 (1996), 315-322.

[GS] V. Guillemin and S. Sternberg, Convexity properties of the moment mapping, Invent. Math. 67 (1982), 491-513.

[Ka] Y. Karshon, Periodic Hamiltonian flows on four dimensional manifolds, dgga/9510004.

[Kn] A. Knudsen, private communication, May 1994.

[Le] E. Lerman, Symplectic cuts, Math. Res. Lett. 2 (1995), 247-258.

[MD] D. McDuff, The moment map for circle actions on symplectic manifolds, J. Geom. Phys. 5 (1988), 149-160. 
[Ok1] A. Okounkov, Log-concavity of multiplicities with an application to characters of $U(\infty)$, and: Newton polyhedrons of spherical varieties, preprints (1994).

[Ok2] _ _ Brunn-Minkowski inequality for multiplicities, Invent. Math., (1996), to appear.

[Pr] A. Prékopa, On logarithmic concave measures and functions, Acta Sci. Math. (Szeged) 34 (1973), 335-343.

Institute of Mathematics, The Hebrew University of Jerusalem, Giv'at RAM, JeRUSALEM 91904, ISRAEL

E-mail address: karshon@math.huji.ac.il 